\title{
Informationsrecht
}

Rainer Kuhlen*

\section{Die Allgemeine Bildungs- und Wissenschaftsschranke im Urheberrecht rückt näher - Kreativität und Innovation werden die Gewinner sein}

DOI 10.1515/iwp-2016-0004

Zusammenfassung: Die obersten Gerichte in Deutschland und in der EU (BGH und EuGH) haben in letzter Zeit einige kreative und der technologischen Entwicklung Rechnung tragende Entscheidungen im Wissenschaftsurheberrecht getroffen - vor allem mit Blick auf die bislang (und auch weiterhin) unzulänglichen und Bildung und Wissenschaft behindernden $\S \S 52$ und 52b. Wirkliche Änderungen im Recht kann aber nur die Politik vornehmen. Änderungen sind nötig: Es bestehen erhebliche Diskrepanzen zwischen urheberrechtlicher Regelung einerseits und der technologischen Entwicklung und den Nutzungserwartungen und -bedürfnissen andererseits. Die EU ist dabei, die seit 2001 bestehende InfoSoc-Richtlinie auf den Prüfstand zu stellen. Ob und vor allem wann Ergebnisse vorliegen werden, ist offen. Die deutsche Politik sollte nicht darauf warten, sondern die im Koalitionsvertrag vereinbarte Allgemeine Bildungs- und Wissenschaftsschranke (ABWS) noch in dieser Legislaturperiode ins Gesetz aufnehmen. Durch eine ABWS wird publiziertes Wissen für Zwecke von Bildung und Wissenschaft zu jeder Zeit, von jedem Ort und über ein beliebiges Medium genehmigungsfrei verfügbar sein. Eine besondere Herausforderung für das Recht besteht darin, einen Neuansatz zur Regelung der Vergütungsfrage zu finden. Anstelle der bislang geforderten individuellen Erhebung einer jeden Nutzung und der Einzelabrechnung sollten sich die Länder mit den Verlagen und der VG-Wort auf pauschalierende Gesamtverträge verständigen. Darüber hinaus sollte das Vergütungsverfahren einer ABWS auch der Besonderheit von Werken Rechnung tragen, die durch öffentliche Finanzierung direkt oder indirekt unterstützt wurden. Deren Nutzung sollte genehmigungs- und vergütungsfrei sein.

*Kontaktperson: Prof.-em. Dr. Rainer Kuhlen, Bogotastraße 4, 14163 Berlin, E-Mail: rainer.kuhlen@uni-konstanz.de, www.kuhlen.name
Deskriptoren: Urheberrecht, Wissenschaft und Technik, Bildungswesen, Gesetz, Vervielfältigung, Vergütung, Nutzung, Europäische Union

\section{A copyright law in favour of education and science is emerging - innovation and creativity will benefit}

Abstract: Supreme courts in Germany and in the EU (BGH and $\mathrm{EuGH}$ ) recently decided creatively in favour of a more liberal understanding of existing exceptions and limitations in copyright law, particularly as far as the use of published works in science and education is concerned. But real change in copyright can only be achieved by the law-makers. The existing and still binding InfoSoc guideline from 2001 can no longer adjust to the technological changes in the last 20 years, let alone to the prevailing needs and expectations of users. The EU has taken a strong stance to tackle the shortcomings of this guideline, particularly as far as science and education are concerned. The outcome to amend the EU copyright regime is not yet certain and will still take some time. Rather than waiting for final results in the EU, politics in Germany (government and parliament) should rapidly implement the Coalition Agreement on a comprehensive exception in copyright law in favour of education and science. According to this exception copying and making published works available to the public is permitted for non-commercial use of scientific research of members in well-defined research groups and for the support of learning and teaching processes. Remuneration for the use of works and library services should be paid on a flat-rate basis by contractual agreement. No remuneration is envisaged for the use of works which have been produced by predominantly publicly financed persons in predominantly publicly financed organizations and projects.

Descriptors: Copyright, Research and technology, Education, Act (legislation), Reproduction, Royalties, Usage, European Union 


\section{L'exception globale au droit d'auteur en faveur de l'éduca- tion et des sciences}

Résumé: Des cours suprêmes en Allemagne et dans l'Union européenne (le Bundesgerichtshof et la Cour de Justice de l'Union européenne) ont récemment pris des décisions créatives en faveur d'une compréhension plus libérale des exceptions et limitations au droit d'auteur, particulièrement en ce qui concerne l'utilisation d'œuvres publiées dans le domaine des sciences et de l'éducation. Mais de réels changements dans la législation ne peuvent être atteints que par la politique. La Directive InfoSoc de 2001, qui est toujours en vigueur, n'est plus adaptée à tous les changements technologiques de ces 20 dernières années et encore moins aux besoins et attentes des utilisateurs. L'UE a pris une position forte pour compenser les insuffisances de cette directive, notamment en ce qui concerne les sciences et l'éducation. Le résultat de ces changements dans l'UE n'est guère certain et le processus prendra encore beaucoup de temps. Plutôt que d'attendre les résultats définitifs dans l'UE, la politique allemande devraient rapidement mettre en œuvre l'accord de coalition qui prévoit une exception globale au droit d'auteur en faveur de l'éducation et des sciences. Selon cette exception, les membres de groupes de chercheurs bien définis auraient l'autorisation de copier des œuvres publiées et de les mettre à la disposition du public pour un usage non-commercial à des fins de recherche scientifique et de soutien aux processus d'apprentissage et d'enseignement. La rémunération pour l'utilisation des œuvres et des services de bibliothèque devrait être payée sur base d'un montant forfaitaire. Par contre, aucune rémunération ne serait exigée pour l'utilisation d'œuvres produites par des personnes principalement financés par des instances publiques ou travaillantes dans des organisations et projets financés majoritairement par l'argent du contribuable.

Descripteurs: Droit d'auteur, Recherche et technologie, Législation, Reproduction, Utilisation, Union européenne, EUCD

„The time has come to move from a negative integration merely based on renewing the restraints of the good functioning of the internal market, to a positive one, including social, cultural and other non-economic considerations."

1 Vgl. Agnès Lucas-Schloetter: Is there a concept of European copyright law? History, evolution, policies and politics and the acquis communitaire. In: Irini Stamatoudi; Paul Torremans (eds.) (2014): EU Copyright Law: A Commentary. Edward Elgar Publishing Limited: Cheltenham, UK, 2014, 7-22; Lucas-Schloetter versteht die aktuelle Aufgabe der EU-Urheberrechtsregulierung nicht mehr primär als Mit-

\section{Zeitgeister}

Die gute Nachricht gleich vorab²: Im Urheberrecht, vor allem im Wissenschaftsurheberrecht bewegt sich einiges zur Zeit noch eher durch Urteile oberster Gerichte wie durch den Bundesgerichtshof (BGH) in Deutschland oder den Europäischen Gerichtshof (EuGH) in der EU. Deren Richter sind ganz offensichtlich willens, die immer noch gültigen, auch Bildung und Wissenschaft betreffenden Vorgaben aus der 2001 verabschiedeten sogenannten InfoSoc-Richtlinie der $\mathrm{EU}^{3}$ zwar nicht zu ignorieren, aber doch nicht mehr ganz so wortwörtlich zu nehmen. Vielmehr schaffen sie mit kreativer Interpretation neue Rechtsrealität.

Ist es so, - wie es die ZEIT 2014 vermutete, als die Richter des Europäischen Gerichtshofs die EU-Richtlinie zur Vorratsdatenspeicherung kassiert hatten -, dass sich die Gerichte zunehmend auch als „Echokammern des Zeitgeistes“ empfinden ${ }^{4}$. Und der „Zeitgeist“ geht derzeit in Richtung einer freizügigeren Nutzung publizierten Wissens in Bildung und Wissenschaft und nicht in Richtung einer Verstärkung des Schutzes der kommerziellen Verwertung von Wissen.

Allerdings ist dieses Zeitgeistverständnis nicht unbedingt die Einschätzung aller der das Recht formulierenden Politiker. Auch konservative Politiker bemühen den Zeitgeist. Allerdings beklagt auf der Konferenz der „Initiative Urheberrecht“ Anfang Dezember 2015 ein nicht unwichtiger unter ihnen, nämlich der EU-Digitalkommissar Günther Oettinger, dass der Zeitgeist in den letzten Jahren „eher in Richtung Piraterie ging“. Das sei nicht mit dem Aufbau eines EU-Binnenmarktes verträglich und dem müsse durch

tel, die Funktionsfähigkeit des internen EU-Marktes zu befördern, sondern um die sozialen, kulturellen, insgesamt: die nicht-kommerziellen Ausprägungen des Umgangs mit Wissen und Information zu unterstützen; das Eingangszitat ist auf S. 16.

2 Ich bedanke mich bei den Mitgliedern der Lenkungsgruppe des Aktionsbündnisses Urheberrecht für Bildung und Wissenschaft (http://www.urheberrechtsbuendnis.de/) für die andauernde Unterstützung und kontinuierliche Informationszufuhr, ohne die meine Arbeit als Sprecher des Aktionsbündnisses nicht möglich wäre und ohne die dieser Artikel nicht hätte entstehen können. Für die Aussagen in diesem Artikel bin ich aber alleine verantwortlich.

3 Directive 2001/29/EC of the European Parliament and of the Council of 22 May 2001 on the harmonisation of certain aspects of copyright and related rights in the information society - http://eur-lex.europa. eu/legal-content/EN/TXT/PDF/?uri=CELEX:32001L0029\&from=EN. 4 Heinrich Wefing: Lex Snowden: Europas Richter schützen die Daten der Bürger vor dem Staat. Aber hilft das gegen die Sammelwut der Amerikaner? DIE ZEIT No. 16, 10. April 2014, S. 1 - http://www.on leihe.de/static/content/zeit_de/20140410/DZ_16_14/vDZ_16_14.pdf Kurz-URL: http://bit.ly/1jdyYQp. 
stärkere Schutzmechanismen gegengesteuert werden. Aber es gibt auch andere Politiker.

So kritisierte (nach heise online) auf der gleichen Konferenz der Bundesjustizminister Heiko Maas <als „Denkfehler“ die Haltung: „Mehr Schutz ist besserer Schutz“. Kulturelles Schaffen baue immer auch auf früheren Nutzungen auf, sodass Felder wie die Public Domain oder die Panoramafreiheit wichtig seien. Noch nie seien so viele hochwertige Inhalte für so viele Menschen verfügbar wie momentan. Im Ergebnis hätten neue Medien immer zu neuem kulturellem Schaffen und mehr Erlösen für Kreative geführt. $^{5}$

Bleiben wir aber noch bei den Gerichten. Fast schon sensationell zu nennen sind z.B. aktuelle Auslegungen eines Paragraphen im Urheberrecht (§ 52b) durch den BGH (nach Vorlage des EuGH). ${ }^{6}$ Diese Norm wurde bei dem sogenannten ersten Korb der Urheberrechtsreform 2003 eingeführt. Dieser Paragraph - entsprechend der Vorgabe in der InfoSoc-Richtlinie (Art. 5, 2, c) - sollte regeln, unter welchen Bedingungen Materialien genutzt werden können, die Bibliotheken aus ihren Beständen digitalisiert haben. Als die Bibliotheken entsprechend dieser Norm gehandelt haben, wurde von Verlagsseite gegen eine nach ihrer Meinung zu weitgehende Nutzung geklagt. Die Klage ging durch alle Instanzen und wurde schließlich vom BGH, im Einklang mit dem EuGH, in so gut wie allen Punkten zurückgewiesen: Bibliotheken dürfen digitalisieren. Nutzer dürfen die Materialien nicht nur einsehen (allerdings nur vor Ort an speziellen „Leseplätzen“ in den Bibliotheken, nicht von extern), sondern auch speichern und zu Hause ausdrucken. Und Verlage dürfen diese Erlaubnisse nicht schon durch ein Verlagsangebot außer Kraft setzen, sondern nur durch einen abgeschlossenen Vertrag.

Dieses BGH-Urteil hat große Irritation oder sogar Panik in der Verlagswelt ${ }^{7}$ hervorgerufen und hat den direkt

\footnotetext{
5 http://www.heise.de/newsticker/meldung/Oettinger-und-die-Urh eberrechtsreform-Der-Zeitgeist-tendiert-zur-Piraterie-3028449.html? wt_mc $=$ nl.ho.2015-12-02.

6 BGH - Urteil vom 16. April 2015 - I ZR 69/11 - Elektronische Leseplätze II - http://juris.bundesgerichtshof.de/cgi-bin/rechtsprechung/ document.py?Gericht=bgh\&Art=en\&nr=72304\&pos $=0 \& a n z=1$. Vorabentscheidungsverfahren des Europäischen Gerichtshofs (EuGH), Urteil vom 11. September 2014 - C-117/13, GRUR 2014, $1078=$ WRP 2012, 1178 - TU Darmstadt/Ulmer

http://curia.europa.eu/juris/document/document_print.jsf?doclang $=$ DE\&text $=$ \&pageIndex $=0$ \&part $=1 \&$ mode $=$ DOC\&docid $=157511 \&$ occ $=$ first\&dir $=\&$ cid $=699928$.

7 Börsenverein kritisiert BGH-Entscheidung zum §52b17. April 2015 „Schwarzer Tag für Forschung und Lehre an Hochschulen,, - http:// www.boersenblatt.net/artikel-boersenverein_kritisiert_bgh-entschei dung_zum_52b.956357.html?nl=newsletter20150419.
}

betroffenen Verlag Eugen Ulmer Ende Oktober veranlasst, dagegen Verfassungsbeschwerde zu erheben. ${ }^{8}$ Ulmer folgt damit der in der Frankfurter Allgemeinen Zeitung vom 13.10.2015 erschienenen Philippika des Heidelberger Philologen Roland Reuß, in der das BGH-Urteil als „Kriegserklärung an das Buch" bezeichnet wurde. ${ }^{9}$

Dass eine solche Beschwerde vom Bundesverfassungsgericht (BverfG) überhaupt angenommen wird, ist eigentlich kaum vorstellbar. Korrigierte der BGH, strikt unter Beachtung der bestehenden Gesetzgebung, doch nur eine bislang zu enge Auslegung einer Norm, die auf der Grundlage der EU-Vorgabe entstanden ist. Entsprechend wurde die BGH-Entscheidung von weiten Kreisen in der Wissenschaft und aus den Bibliotheken begrüßt und auch als wichtiger Schritt in Richtung einer Allgemeinen Bildungs- und Wissenschaftsschranke verstanden. ${ }^{10}$

\section{EU InfoSoc-Richtlinie2001 und Internetwelt 2015}

Offensichtlich ist für jedermann, dass die alte InfoSocRichtlinie der EU weder für die EU selbst noch für die einzelnen Länder weiterhin richtungsweisend sein kann. Und solange die Politik noch zögerlich war, daran etwas zu ändern, sind Kreativität der Auslegung und die stärkere Berücksichtigung der Entwicklungen und der Bedürfnisse der durch das Urheberrecht Betroffenen dringend erforderlich.

Die Internet-Welt ist heute gegenüber 2001 eine ganz andere geworden. Das gilt nicht nur für die allgemeinen Publikumsmärkte, auf denen für so gut wie jedermann der Zugriff auf weltweite multimediale Informations- und Kommunikationsdienste eine Selbstverständlichkeit geworden ist. Aber auch die gegenwärtigen Wissensproduktions-, Publikations- und Nutzungsformen in Bildung und Wissenschaft waren vor 15 Jahren noch unvorstellbar.

Nur einige Beispiele für Diskrepanzen zwischen urheberrechtlicher Regelung einerseits und der technologi-

\footnotetext{
8 http://www.buchreport.de/nachrichten/nachrichten_detail/da tum/2015/10/21/ulmer-erhebt-verfassungsbeschwerde.htm. 9 FAZ 13.10.2015, Nr. 337, S. 11.

10 Vgl. Rainer Kuhlen: Eine Entscheidung auf dem Weg zur Wissenschaftsschranke durch den BGH: Ein Schritt in die richtige Richtung, alles andere als ein schwarzer Tag für Forschung und Lehre http://www.urheberrechtsbuendnis.de/stellungnahme-kuhlen-201504-20-bgh-52b.pdf; Eric Steinhauer: EuGH-Urteil zu elektronischen Leseplätzen stärkt Wissenschaftsurheberrecht. GRUR-Prax 2014, 471 (über beck-online).
} 
schen Entwicklung und den Nutzungserwartungen und -bedürfnissen andererseits:

1. Noch 2001 wurden selbst von technischen Experten wie universitären Rechenzentrumsleitern elektronische Bibliotheken als nicht realisierbar eingeschätzt. Mehr als etwa 1000 Büchern könnten aus technischen und Kostengründen gar nicht digitalisiert und gespeichert werden. Heute dürfen es auch eine Million und mehr sein - wenn nicht das bestehende Urheberrecht in der EU dem oft unüberwindbare Hindernisse entgegenstellen würde - anders als in den USA, wo jüngst nach ca. zehnjährigem Streit das zuständige Gericht die Millionen-Digitalisierung im Rahmen von Google-Books für rechtens erklärt hat. ${ }^{11}$

2. Die gegenwärtigen Technologien haben nicht nur Aufbau und Nutzung riesiger Datenbestände möglich gemacht. Vielmehr entsteht, wiederum vor allem in den USA, durch Big Data und durch Text Data Mining (TDM) ein ganz neues Forschungsparadigma: Die Daten sind da. Aus toten Daten wird relevante Information abgeleitet, die zu neuem Wissen verdichtet werden kann. Neues Wissen kann durch Auswertung entstehen wenn denn der Zugriff auf die Daten erlaubt ist. Viele Datenbanken sind in kommerzieller Hand, und die Rechteinhaber wollen darauf beharren, dass für das Data Mining auch für Zwecke der Forschung noch einmal Lizenzen gezahlt werden müssen - zusätzlich zu den bislang schon von den Bibliotheken bezahlten Lizenzen für das Lesen. Ausnahmeregelungen zugunsten des Data Mining für Forschung sind im Urheberrecht in der EU so gut wie nicht vorhanden. Die Technologie ist da, das Urheberrecht mit dem Schutz der Verwertungsrechte ermöglicht sie bislang nicht, sondern behindert sie - nur England ist vorgeprescht. In den USA ist TDM ohnehin weitgehend freizügig als fair use möglich.

3. Der Zugriff auf das weltweite Wissen, einschließlich des Zugriffs auf die Bestände der eigenen Bibliothek vom Arbeitsplatz aus, wo immer der auch gerade ist, wird heute nicht als Privileg, sondern als technologische Selbstverständlichkeit empfunden. So gut wie jeder (nicht nur Forscher und Lehrer, sondern auch die Studierenden) verfügt über Computer und Netzanschluss - das gegenwärtige Urheberrecht verlangt aber, dass man in die Räume der Bibliotheken gehen muss, um an speziell dafür eingerichteten „Leseplät-

11 Vgl. http://phys.org/news/2015-10-appeals-court-google-book-sc anning.html - Eine Supreme Court Entscheidung steht allerdings noch aus. zen“ digitalisierte Werke aus den Beständen der Bibliothek einsehen zu können. Das hält jetzt sogar die EUKommission in ihrem vorab schon Anfang November 2015 bekannt gewordenen Bericht ,Towards a modern, more European copyright framework“ angesichts der technologischen Entwicklung nicht mehr für zeitgemäß.12

4. Wissenschaft, aber auch zunehmend E-Learning organisieren sich grenzüberschreitend. Diese grenzüberschreitende Nutzung von Bibliotheksbeständen funktioniert auch ganz gut. So bietet z.B. die Euregio Oberrhein Nutzern Ausleihmöglichkeiten von Büchern in den Bibliotheken in Basel, Freiburg/B und Straßburg mit einem Ausweis an - wohlgemerkt von Büchern in gedruckter Form. Für die grenzüberschreitende Nutzung von eBooks gibt es keine urheberrechtlich legitimierte Regelung. eLending war 2001 kein Thema.

Das Recht bewegt sich langsam und konnte bislang nur sehr unzureichend auf solche Veränderungen reagieren. Da sollte es schon eine Hilfe sein, dass sich der Bundesgerichtshof daran gemacht hat, einige der Schäden zu reparieren, die das existierende Urheberrecht für Bildung und Wissenschaft angerichtet hat, oder zumindest Missverständnisse durch Rechtsfortbildung aufzulösen. Von „Kriegserklärung“ (Reuss, s.o.) kann keine Rede sein, eher handelt es sich um den Versuch der Befriedung, nämlich die in Bildung und Wissenschaft Tätigen und die Bibliotheken von Unsicherheit zu befreien: Darf man z. B. digitale Materialien der Bibliotheken zur Weiternutzung abspeichern oder setzt man sich dem Verdacht einer kriminellen Handlung aus? Nein, jetzt ist es klar: man darf. Dürfen Studierende die von ihren Lehrenden in elektronischen Semesterapparaten bereitgestellten Materialien nur im Unterricht wahrnehmen (so heißt es im Gesetz) oder dürfen sie das auch für Nach- und Vorbereitung der Kurse verwenden? Ja, sie dürfen - der BGH ist dieser Ansicht, da sonst der entsprechende Paragraph (§ 52a) keinen Sinn mache.

12 Communication from the Commission to the European Parliament, the Council, the European Economic and Social Committee and the Committee of the Regions: Towards a modern, more European copyright framework (angekündigt für Dezember 2015 - die Anfang November vorab geleakte Version unter: https://drive.google.com/fil e/d/0B6d07lh0nNGNaXFzUFBPaE0tYOE/view). 


\section{Der Gesetzgeber übernimmt die Initiative}

Allerdings, auch die Gerichte stoßen an ihre Grenzen und können letztlich nur auslegen, was die Gesetze vorgeben. Wirkliche Veränderungen im Gesetz kann nur die Politik vornehmen. Und sie ist dabei - sowohl auf der europäischen Ebene als auch in Deutschland. Die Politik scheint willens zu sein, selber wieder die Initiative zur Gestaltung des Urheberrechts zu übernehmen.

\subsection{Erste Ansätze zu einem zeitgemäßen Urheberrecht in Deutschland}

Dass die deutsche Politik es kann, hat sie vor zwei Jahren erfolgreich demonstriert: In Deutschland hat der Bundestag mit Wirkung Anfang 2014 zwei nicht unwichtige Gesetzesvorhaben auf den Weg gebracht: Zum einen eine Regelung des Zweitveröffentlichungsrechts (in Ergänzung zu der kommerziellen Publikation) zugunsten der freien Nutzung und zum andern eine Regelung für den Umgang mit verwaisten und vergriffenen Werken ${ }^{13}$.

Beide Normen sind zwar durchaus verbesserungsbedürftig (z. B. gilt das Zweitveröffentlichungsrecht nicht für die normale, also nicht drittmittelgefördert Hochschulforschung ${ }^{14}$ und die an sich politisch erwünschte Massendigitalisierung von Beständen z. B. aus Bibliotheken ist durch komplizierte Regelungen kaum zu erreichen) - aber immerhin!

Wissenschaftliche Autoren haben damit einen Teil der Freiheit gegenüber den kommerziellen Verlagsverträgen zurück gewonnen, und die Regelung für vergriffene Werke

13 Beide Normen wurden zusammen verabschiedet: Gesetz zur Nutzung verwaister und vergriffener Werke und einer weiteren Änderung des Urheberrechtsgesetzes vom 1. Oktober 2013 http://www.bundes gerichtshof.de/SharedDocs/Downloads/DE/Bibliothek/Gesetzesmate rialien/17_wp/UrheberR_verwaiste_Werke_BReg/bgbl.pdf?_blob=p ublicationFile

14 Vgl. Rainer Kuhlen: Manifestierung der Dreiklassengesellschaft [zum Zweitverwertungsrecht]. Spektrum.de (Spektrum der Wissenschaft) 19.9.2013 - http://www.spektrum.de/alias/urheberrecht/man ifestierung-der-dreiklassengesellschaft/1207757; Interdependenzen zwischen Informationsethik und politischem Handeln - am Beispiel einer kontroversen Urheberrechtsregulierung (Zweitverwertungsrecht). In: Zeitschrift für Politikwissenschaft (ZPol), Sonderband Ethik und Politikmanagement, 2014, 193-221, Preprint: http://www.kuh len.name/MATERIALIEN/Publikationen2014/interdependenzen-infor mationsethik-urheberrecht-PDF.pdf. ist über die europäische Verwaiste-Werke-Richtlinie hinausgegangen und hat sich als Erfolgsmodell erwiesen.

\subsection{Reform der europäischen Urheberrechtsregulierung}

Die EU-Kommission hat Ende 2013 den Weg in die Öffentlichkeit gesucht, um die seit langem geforderte Urheberrechtsreform anzustoßen - nicht zuletzt mit Blick auf die sogenannte InfoSoc-Richtlinie von 2001. Zwischen Dezember 2013 und März 2014 wurde eine öffentliche Befragung zur Reform der EU-Urheberrechtsregeln durchgeführt, die mehr als 9500 Antworten aus den verschiedenen, vom Urheberrecht betroffenen Gruppen (z. B. Autoren, Nutzer, Verleger, Provider, Informationsvermittler, Verwertungsgesellschaften) erhalten hat. ${ }^{15}$ Die Kommission hat diese Befragung detailliert ausgewertet. ${ }^{16}$

In diesem Zusammenhang ist vor allem Kapitel IV Limitations and exceptions in the single market einschlägig und dort die Abschnitte 5. Teaching (Questions 42 to 46) und 6. Research (Questions 47-49).

Deutlich wurde bezüglich Bildung der Bedarf angemeldet „for a broad exception for non-commercial use of protected works in educational contexts: they believe that the exception should not be limited to educational establishments, teachers and students but should cover all educational activities (including non-formal education) and should not give rise to compensation. According to respondents, the exception should be technologically neutral, to cover face-to-face teaching and online education.“(53f.)

Im Bereich Forschung wurden nicht zuletzt die Restriktionen der kommerziellen Verwertung als Hindernis beklagt: „this situation is particularly difficult to accept in the case of publicly-funded research. They [researchers RK] believe that publications which present the results of publicly funded research should always be made available without restriction." Ausnahmebestimmungen zugunsten von Bildung und Wissenschaft sollten in allen EU-Ländern verpflichtend sein.

15 Alle Antworten sind vollständig dokumentiert unter http://ec.eur opa.eu/internal_market/consultations/2013/copyright-rules/index_ en.htm.

16 European Commission (2014). Directorate General Internal Market and Services. Directorate D - Intellectual property. D1 - Copyright: Report on the responses to the Public Consultation on the Review of the EU Copyright Rules. July 2014 - http://ec.europa.eu/internal_mar ket/consultations/2013/copyright-rules/docs/contributions/consulta tion-report_en.pdf. 101 pp. 
Als weiteren Schritt hat die EU-Kommission am 6. Mai 2015 ein Planungspapier „A Digital Single Market Strategy for Europe ${ }^{\text {“17 }}$ vorgelegt. In dieser Strategie steht nach wie vor, und in der Tradition der InfoSoc-Richtlinie von 2001, das ökonomische Verwertungsinteresse von Wissen und Information im Vordergrund. Allerdings sollen die umfänglichen und in der Praxis eher verwirrenden Ausnahmebestimmungen der alten Richtlinie (Ausnahmen von den an sich als exklusiv angesehenen Rechten der Urheber und Verwerter) EU-weit harmonisiert und aktualisiert werden. Das ist alles noch sehr vage, aber lässt Spielräume, die genutzt werden sollten, wenn es dann einmal um die Umsetzung der bisherigen Planungspapiere geht.

Auch das EU-Parlament, das aber in der EU keine Gesetzesinitiative hat, hat sich auf seine Position zur Urheberrechtsreform verständigt. Grundlage dafür sollte ein Bericht des Parlaments mit Forderungen an die Umsetzung bzw. Neugestaltung der Richtlinie von 2001 (der „InfoSocRichtlinie“) sein. Dazu wurde Julia Reda (Abgeordnete der Piratenpartei im Europäischen Parlament) zur Berichterstatterin ernannt. Der von ihr dem Committee for Legal Affairs vorgelegte Entwurf (der sogenannte Reda-Bericht) ${ }^{18}$ wurde am 9. Juli vom EU-Parlament mit einigen Änderungen $^{19}$ angenommen. ${ }^{20}$ Dies ist, wie oben angedeutet, kein Gesetzentwurf, sondern nur eine Zielvorstellung des Parlaments, die aber von der Kommission nicht einfach ignoriert werden kann.

In dem in Abschnitt 2 unter Top. 3 schon erwähnten Strategieplan der EU-Kommission „Towards a modern, more European copyright framework" nimmt sich die Kommission vor, Schrankenregelungen der alten EU-

17 Communication from the Commission to the European Parliament, the Council, the European Economic and Social Committee and the Committee of the Regions: A Digital Single Market Strategy for Europe. $\{\operatorname{SWD}(2015) 100$ final $\}$. Brussels, 6.5.2015 $\operatorname{COM}(2015) 192$ final - http://ec.europa.eu/priorities/digital-single-market/docs/ds m-communication_en.pdf

18 DRAFT REPORT on the implementation of Directive 2001/29/EC of the European Parliament and of the Council of 22 May 2001 on the harmonisation of certain aspects of copyright and related rights in the information society (2014/2256(INI)) Committee on Legal Affairs Rapporteur: Julia Reda -https://pub.juliareda.eu/copyright_evaluation_ report.pdf.

19 Der Rechtsausschuss des EU-Parlaments (Committee for Legal Affairs) hatte sich mit 556 (!) Kommentaren und Änderungsvorschlägen aus allen Fraktionen des Parlaments auseinanderzusetzen.

20 European Parliament resolution of 9 July 2015 on the implementation of Directive 2001/29/EC of the European Parliament and of the Council of 22 May 2001 on the harmonisation of certain aspects of copyright and related rights in the information society (2014/2256(INI) -http://www.europarl.europa.eu/sides/getDoc.do?type=TA\&referenc $\mathrm{e}=$ P8-TA-2015-0273\&language $=$ EN.
Richtlinie im Lichte der heutigen technologischen Realitäten neu zu bewerten (,need to be reassessed in the light of today's technological realities“). Dies gelte vor allem für die Regelungen „closely related to education, research and access to knowledge." Das klingt vielversprechend.

Die Umsetzung all dessen in der EU verzögert sich. Ursprünglich, bei Aufnahme ihrer Arbeit 2014, hatte sich die Kommission vorgenommen, bis Ende 2015 entscheidungsfähige Vorschläge dem europäischen Parlament und dem Rat vorzulegen. Dieser Termin ist verstrichen. Vermutlich wird die EU nicht ein Gesamtpaket vorlegen, sondern schrittweise die einzelnen Bereiche abarbeiten. Speziell für Bildung und Wissenschaft ist kaum vor Ende 2016 mit entscheidungsreifen Vorschlägen zu rechnen.

Aber diese Verzögerung sollte die Politik in Deutschland nicht mitmachen. Die deutsche Politik, federführend das Bundesministerium der Justiz und für Verbraucherschutz (BMJV), muss nicht auf die EU warten. Julia Reda sah auf einem Workshop des Aktionsbündnisses am 15. Oktober diesen Jahres (vgl. den Bericht in diesem Heft) in Berlin für Deutschland eine Chance, durch rasche Verabschiedung einer Allgemeinen Bildungs- und Wissenschaftsschranke (ABWS) ein Vorbild nicht nur für die EUEntwicklung, sondern auch für die in anderen Ländern zu geben. Der Einfluss Deutschlands auf die EU-Entwicklung und auf die Urheberrechtsentwicklung in den anderen EUStaaten könne gar nicht groß genug eingeschätzt werden. Politiker in der EU scheinen geradezu auf eine Initiative aus Deutschland zu warten.

\section{Für Bildung und Wissenschaft eine umfassende Urheberrechtsregelung}

Bildung und Wissenschaft warten auch - seit bald 15 Jahren auf Verbesserungen im Urheberrecht zu ihren Gunsten. Die bisherigen Schrankenregelungen aus dem ersten und zweiten Korb der Urheberrechtsreform (2003 und 2008) zu belassen und nur geringfügig zu verbessern, wird nicht den Durchbruch bringen. Erwartet wird eine generelle Reform mit einer Allgemeinen Bildungs- und Wissenschaftsschranke (ABWS).

Politisch sind in Deutschland die Weichen dafür an sich schon gestellt. Im Koalitionsvertrag der jetzigen Bundesregierung hat man sich auf die Einführung einer ABWS verbindlich verständigt. Es heißt im Vertrag nicht „wir wollen“, sondern „wir werden“. Und in der Tat: Deutet man die Zeichen aus dem BMJV richtig, dann sollte spätestens im Frühjahr 2016 ein Referentenentwurf einer 
ABWS vorliegen und in die interministerielle Abstimmung gehen. Die Allgemeine Bildungs- und Wissenschaftsschranke nimmt im Urheberrecht Fahrt auf.

\subsection{Vorleistungen}

Natürlich wird alles darauf ankommen, wie am Ende diese ABWS im Detail gestaltet und in welcher Form sie schließlich vom Bundestag beschlossen werden wird. Was soll das federführende Ministerium, das Bundesministerium der Justiz und für Verbraucherschutz, in seinen Referentenentwurf einer ABWS hineinschreiben? Und wird sich das Reformvorhaben in den Ausschüssen des Bundestags gegen den starken Druck des Verlags-Lobbying behaupten können? Keine einfache Aufgabe für die Politik! Es liegt aber Einiges an Vorleistungen vor. ${ }^{21}$

- Im europäischen Kontext hat die Wittem-Gruppe Vorstellungen für einen European Copyright Codevorgelegt und dabei eine weitgehend uneingeschränkte Nutzung urheberrechtlich geschützter Materialien für Wissenschaft und Bildung vorgeschlagen ${ }^{22}$. Begründet wird diese Nutzung nicht zuletzt durch einen Hinweis auf das Menschenrecht der Informationsfreiheit (freedom of expression and information). Welcher Art die Nutzungen sind und in welcher Extension sie in Anspruch genommen werden können, wird nicht weiter spezifiziert. Das bedeutet, dass hierdurch jede Nutzung begünstigt wird, die aus wissenschaftlichen Gründen (purposes) erfolgt. Einschränkungen des Umfangs der Nutzung, wie sie in dem deutschen $\S 52 \mathrm{a}$ vorgesehen sind, der leider oft schon als Wissenschaftsschranke bezeichnet wird, gibt es im WittemVorschlag nicht: „The following uses for the purpose of freedom of expression and information are permitted without authorisation, but only against payment of remuneration and to the extent justified by the purpose of the use:

(a) use of single articles for purposes of internal reporting within an organisation;

(b) use for purposes of scientific research.”

21 Die Ausführungen zu diesen Vorleistungen stützen sich ab auf Formulierungen aus: Rainer Kuhlen: Wie umfassend soll/darf/muss sie sein, die allgemeine Bildungs- und Wissenschaftsschranke? In: Zeitschrift für Geistiges Eigentum/Intellectual Property Journal, Volume 7, 2015, Number 1, pp. 77-125, speziell aus Kap. 3.

22 Wittem Group, The Wittem Project. European copyright code. April 2010 - www.copyrightcode.eu; vgl. R. Kuhlen, Richtungsweisend oder eine verpasste Chance? Der Copyright-Code des Wittem Projekts. In: Information Technology and E-Commerce Law (JIPITEC) 2/2011, S.18-25.
- Ein umfängliche Studie mit einem konkreten Normvorschlag für eine ABWS hat die Berliner HU-Professorin Katharina de la Durantaye vorlegt. ${ }^{23}$ Sie hat in einem vom Bundesministerium für Bildung und Forschung (BMBF) finanzierten Forschungsauftrag ausgelotet, was entsprechend dem gegenwärtigen (auch europarechtlichen) Stand der Gesetzgebung und Rechtsprechung möglich ist. Diese Studie folgt entsprechend der Vorgabe des BMBF dem acquis communitaire und geht nicht auf weitergehende Perspektiven ein.

- Das Aktionsbündnis, das vor etwa sechs Jahren die Diskussion um eine ABWS angestoßen hatte, verfolgt mit seinem aktuellen Norm-Vorschlag einen anderen, strikt teleologischen, also am Zweck von Bildung und Forschung orientierten Weg. ${ }^{24}$ Was an publiziertem Wissen für den Zweck von Bildung und Forschung erforderlich ist, sollte ohne weitere Einschränkung genehmigungsfrei eingesehen und genutzt werden. Ein Wegfall der jetzigen im Gesetz verteilten Schrankenregelungen wäre auch ein Beitrag zur erwünschten Verschlankung des jetzigen komplexen Urheberrechts.

- Die Allianz der Wissenschaftsorganisationen hat das Konzept einer umfassenden ABWS 2010 aufgegriffen und sich dabei stärker an den bisherigen Formulierungen des Urheberrechtsgesetzes angelehnt, insbesondere an $\S 52 \mathrm{a}^{25}$. Allerdings sollte es bei dem Vorschlag keineswegs nur um eine Erneuerung von $\S 52$ a gehen. Vielmehr sollten in ihm über eine neue Norm $\S 45 \mathrm{~b}$ die

23 Katharina de la Durantaye: Allgemeine Bildungs- und Wissenschaftsschranke. Verlagshaus Monsenstein und Vannerdat OHG 2014 - herunterladbar unter: http://durantaye.rewi.hu/doc/Wissen schaftsschranke.pdf.

24 Vgl. vor allem die beiden ersten Absätze: „1) Zulässig ist die Vervielfältigung und öffentliche Zugänglichmachung eines veröffentlichten Werkes für nicht kommerzielle Zwecke a) wissenschaftlicher Forschung für Mitglieder in formal eindeutig bestimmten Forschungsgruppen oder b) der Lehr- und Lernprozesse von Lehrveranstaltungen an Bildungseinrichtungen. Satz 1 gilt auch für Zwecke der Bestandserhaltung durch Einrichtungen wie öffentlich finanzierte Bibliotheken, Archive, Dokumentationen und Museen. Satz 1 gilt auch für die wissenschaftliche Forschung und Lehren und Lernen unterstützende Leistungen von in Satz 2 erwähnten Vermittlungsinstitutionen.

(2) Für die Nutzung von Werken, die in öffentlich finanzierten Umgebungen unter Beteiligung von öffentlich finanzierten Personen erstellt wurden, ist keine Vergütung vorgesehen.“ http://www.urheber rechtsbuendnis.de/abws-text-2014-12.html.de

25 Vgl. Allianz der Wissenschaftsorganisationen: Neuregelung des Urheberrechts: Anliegen und Desiderate für einen Dritten Korb vom 9.7.2010, hier der Vorschlag für einen $\S 45 \mathrm{~b}$ Wissenschaftlicher Gebrauch und Bildung, S. 8-10.(http://www.wissenschaftsrat.de/down load/archiv/Allianz_Desiderate_UrhG.pdf). 
bisherigen Regelungen der $\S \S 46,47,51,52 \mathrm{a}, 52 \mathrm{~b}, 53$, 53 a zusammengeführt werden. Die Allianz verfolgte in der Zusammenfassung bestehender Normierungen einen synoptischen pragmatischen Ansatz.

- Auch die Kultusministerkonferenz (KMK) ist in ihrem Positionspapier von 2009 der Auffassung, „dass die de lege lata in den $\S \S 52 \mathrm{a}, 52 \mathrm{~b}$ und 53a normierten Schrankenregelungen de lege ferenda nicht durch eine Synopse wie bei der Allianz, sondern durch eine generalklauselartig gefasste einheitliche Schrankenregelung für die Bereiche Bildung und Wissenschaft zu ersetzen sind“.

Die Kultusministerkonferenz schlug dafür einen erneuerten $\S 52$ a vor, durch den „die immer kleinteiliger und in der Praxis kaum noch handhabbar gewordenen Schrankenregelungen ersetzt“ würden und der nach Einschätzung der Kultusministerkonferenz ebenfalls „mit der EU-Richtlinie 2001/29/EG vom 22.05.2001 konform" sei. Allerdings sollen in diesem Vorschlag die Rechte der Vervielfältigung, Verbreitung und öffentlichen Zugänglichmachung eines veröffentlichten Werkes nicht den Nutzern direkt zugestanden werden, sondern „öffentlichen Einrichtungen, denen Aufgaben in Bildung, Wissenschaft und Kultur übertragen sind".

- Auch der Deutsche Bibliotheksverband hat im Juni 2014 einen Vorschlag für eine ABWS vorgelegt ${ }^{26}$, der derzeit überarbeitet wird.

- Und schließlich wird derzeit im Auftrag des Bundesministeriums für Bildung und Forschung von Juristen der Universität Göttingen (federführend Prof. Spindler) eine Studie angefertigt, welche die ökonomische Relevanz und die ökonomischen Konsequenzen einer $A B W S$ untersuchen soll.

- Und natürlich liegen aus der Verlagssicht viele Stellungnahmen vor, in denen eine ABWS für überflüssig und als ganz und gar schädlich abgelehnt wird.

\subsection{Zielvorstellungen - Prüfbausteine}

Ein juristisch stabiler Vorschlag für eine ABWS wird in erster Linie von der Politik erwartet - in einem ersten Vorschlag durch einen Referentenentwurf des BMJV.

Das Aktionsbündnis Urheberrecht für Bildung und Wissenschaft beharrt in seinen Bemühungen um eine ABWS derzeit weniger auf dem eigenen Normvorschlag (s.

26 http://www.bibliotheksverband.de/fileadmin/user_upload/DBV/ positionen/2014_06_25_Stellungnahme_Wissenschaftsschranke.pdf. oben), sondern hat eine Liste von Zielvorstellungen bzw. Prüfbausteinen aufgestellt und in die politische Planung eingebracht. ${ }^{27}$ Hier einige Hinweise aus dieser Liste, was aus der Sicht der in Bildung und Wissenschaft Tätigen durch eine ABWS erreicht werden sollte:

- Durch eine ABWS wird publiziertes Wissen für Zwecke von Bildung und Wissenschaft zu jeder Zeit, von jedem Ort und über ein beliebiges Medium genehmigungsfrei verfügbar sein.

- Regelungen in der ABWS haben Vorrang vor Vertragsvorgaben aus der Informationswirtschaft und können daher nicht durch Verträge außer Kraft gesetzt werden.

- Die ABWK ersetzt das bisherige komplizierte System der verschiedenen, Bildung und Wissenschaft betreffenden Schrankenregelungen.

- Die Regelungen der ABWS für die Nutzung von publiziertem Wissen sind anders als im bestehenden Recht so festgelegt, dass jedermann - Wissenschaftler, Lehrende und Lernende -verstehen kann, welche Nutzungen erlaubt sind.

- Die ABWS privilegiert auch die traditionellen und die elektronisch verteilt und kollaborativ organisierten Lehr- und Lernprozesse sowie die Formen selbstbestimmten und selbstorganisierten Lernens.

- Die ABWS ist technikneutral und offen für technologische Weiterentwicklungen, wie z.B. aktuell durch Verfahren des Text Data Mining. Entsprechende Werkzeuge (Auswertungsalgorithmen) dürfen frei zur Anwendung kommen bei Material, das für den Zugang bereits lizenziert wurde.

\subsection{Herausforderung: Vergütung - immer ein Muss?}

Eine besondere Herausforderung wird es sein, einen Neuansatz zur Regelung der Vergütungsfrage zu finden. Bislang gilt in Recht und Politik, dass in Bildung und Wissenschaft so gut wie jede durch eine Schrankenregelung erlaubte Nutzung publizierter Werke zu einer Entschädigung der Rechteinhaber, also zu einer Vergütung führen müsse ${ }^{28}$. Zudem müsse, damit es dabei gerecht bleibe, jede einzelne Nutzung registriert und dann die Vergütung auf dieser individuellen Basis geleistet werden.

27 Was eine Allgemeine Bildungs- und Wissenschaftsklausel (ABWK) leisten soll. Zielvorstellungen, Prüfbausteine. November 2015 - Text unter http://www.urheberrechtsbuendnis.de/

28 Ausnahmen von der Vergütungsverpflichtung gibt es im Urheberrecht. Z. B. muss für die Wahrnehmung des Zitatrechts (geregelt in $\S 51$ UrhG) keine Vergütung entrichtet werden. 
Die Politik wird aber wohl kaum an den auf dem Workshop vorgestellten Ergebnissen einer Machbarkeitsstudie an der Universität Osnabrück vorbeikommen ${ }^{29}$, durch die Kennzahlen für Kosten, Aufwand und Workflows bei der Einzelerfassung und Abrechnung von Lehrmaterialien (entsprechend den Vorgaben von §52a UrhG und der Rechsprechung des BGH dazu ${ }^{30}$ ) ermittelt wurden. Das Fazit ist eindeutig: ${ }^{31}$

Sowohl für die Lehrenden als auch für die Studierenden ist der Aufwand für die Einzelerfassung nicht zumutbar und nicht zu rechtfertigen. Sie wirkt abschreckend und führt tatsächlich zu einem Rückgang bei der Bereitstellung von Materialien in der Lehre und damit auch zu Einbußen in deren Qualität. Auch könnte die individuelle Erhebung der Nutzung und damit die Transparenz der verwendeten Werke für Dritte als Einschränkung von Wissenschaftsfreiheit empfunden werden. Sinnvoller wäre die Rückkehr zur Pauschalvergütung über Gesamtverträge. Dies sollte die leichtere Übung sein.

Aber die Herausforderung, für die Nutzung urheberrechtlich geschützter Materialien eine Vergütungsregelung zu finden, ist noch größer. Anstrengender, mutiger, aber auch zukunftsweisender ist es, die generellere Frage zu beantworten, ob in Bildung und Wissenschaft in jedem Fall eine Vergütungsgarantie durch das Urheberrecht gegeben werden muss.

Vergütung gehört sozusagen zu den „Heiligen Kühen“ des Urheberrechts. Selbstverständlich haben Kreative auf den Publikumsmärkten, in der Belletristik, in der Musik oder in den Medien einen Rechtsanspruch auf angemessene Vergütung für ihre Produktionen. Sie müssen i.d. R. von diesen Einnahmen ihren Lebensunterhalt bestreiten.

Aber in der Wissenschaft, vor allem in der öffentlich finanzierten Wissenschaft, ist es anders. Die dort Kreativen werden bereits für ihre Tätigkeiten, auch für das Publizieren, durch das Gehalt bezahlt, und ihnen wird jedwede (technische, personelle, räumliche) Infrastruktur für ihr Schaffen bereitgestellt. Monetäre Belohnung ist zudem nicht das primäre Motiv für Kreativität und Publikation. Sichtbarkeit und Reputation ist die Anerkennungswährung (die sich dann auch oft in monetäre Anerkennung zur

29 Pilotprojekt zur Einzelerfassung der Nutzung von Texten nach § 52a UrhG an der Universität Osnabrückhttps://www.virtuos.uni-os nabrueck.de/forschung/projekte/pilotprojekt_zum_52a_urhg.html.

30 BGH Urteil vom 28. November 2013 Az. I ZR 76/12 (Meilensteine der Psychologie) - https://openjur.de/u/660333.html.

31 Vgl. May-Britt Kallenrode: Im Prinzip ja. Zur Einzelabrechnung nach §52a UrhG (Vortragsfolien) - http://www.urheberrechtsbuend nis.de/docs/ws2015/kallenrode.pdf - Website des Modellversuchs: ht tp://www.virtuos.uni-osnabrueck.de/Projekte/Pilot52a
Verbesserung der professionellen Situation einwechseln lässt).

Abgesehen davon erhalten die Autoren im wissenschaftlichen Publikationswesen tatsächlich wenn überhaupt dann, bis auf wenige Ausnahmen, nur ein spärliches Anerkennungshonorar, kaum eine der Leistung angemessene Vergütung. Für Zeitschriftenartikel ist so gut wie nie von den Verlagen eine Vergütung vorgesehen. ${ }^{32}$

Insbesondere sollte das Vergütungsverfahren einer ABWS auch der Besonderheit von Werken Rechnung tragen, die durch öffentliche Finanzierung direkt oder indirekt unterstützt wurden. Das Argument der öffentlichen Finanzierung von Werken und von öffentlich finanzierten Forschern ist bei der Frage der Vergütung bislang kaum berücksichtigt worden. Sollte es aber, denn auch hierdurch wird deutlich, dass die Vergütungsfrage sich in Bildung und Wissenschaft ganz anders stellt als auf den Publikumsmärkten. Fällt in diesen Fällen eine Vergütung an die Autoren fort, sollten die entsprechenden Werke genehmigungs- und vergütungsfrei nutzbar sein.

Ohnehin deutet die Open-Access-Entwicklung, befördert durch das stetige Anwachsen auch kommerzieller Open-Access-Zeitschriften und durch eine erkennbare Tendenz zur Mandatierung von Open-Access-Publikationen, darauf hin, dass Vergütung im Bereich Bildung und Wissenschaft bis auf wenige Ausnahme nicht die entscheidende Rolle spielt bzw. gar nicht mehr passiert. Muss Vergütung dann weiter im Recht verbindlich geregelt werden?

\section{Fazit}

Entscheidend für das Urheberrecht auch in Deutschland wird die Entwicklung in der EU sein. Allerdings ist es derzeit noch nicht absehbar, dass die Politik (in erster Linie also der EU Rat und dann in der Realisierung die EU-Kommission und hoffentlich dann stärker beteiligt das EU-Parlament) sich auf ein genuin europäisches, also EU-weit gültiges, nicht nur harmonisiertes EU-Urheberrecht verständigt. Bis auf weiteres ist das Urheberrecht eine jeweils nationale Aufgabe, allerdings mit immer stärker werdenden Vorgaben der EU - gleichermaßen der EU-Rechtsetzung und der immer wichtiger und einflussreicher werdenden Entscheidungen des Europäischen Gerichtshofs (EuGH).

32 Eine der wenigen Ausnahmen sind Arbeiten in Zeitschriften der Rechtswissenschaft, die von einigen Verlagen vergütet werden. Liegt es daran, dass Juristen auf Vergütung im Urheberrecht bestehen? 
Die Aufgabe, ein zeitgemäßes Urheberrecht und in ihm ein Kreativität und Innovation unterstützendes Wissenschaftsurheberrecht zu schaffen, bleibt also die Herausforderung an die deutsche Politik. Mit dem Koalitionsvertrag der jetzigen Regierung hat sie diese Herausforderung angenommen, Man kann nur hoffen, dass die Politik, zuerst das BMJV und dann der Bundestag, den Mut zu einer wirklichen Reform findet und nicht nur an bestehenden Normen herumdoktert. Der Politik wird bewusst sein, dass es von den meisten in Bildung und Wissenschaft Tätigen (vermutlich auch vom überwiegenden Teil der Bevölkerung) für eigentlich selbstverständlich gehalten wird, dass das mit öffentlichen Mitteln geförderte Wissen für jeden frei zugänglich sein soll und frei genutzt werden kann. Und ebenso wird sie erkannt haben, dass Kreativität in der Wissenschaft und Innovationsfähigkeit in der Wirtschaft umso höher sein wird, je freier der Umgang mit Wissen und Information ist. Es besteht eine realistische Chance, dass eine Allgemeine Bildungs- und Wissenschaftsschranke auf einen guten Weg gebracht werden wird.

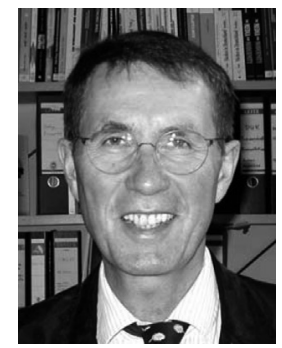

Prof.-em. Dr. Rainer Kuhlen

Bogotastraße 4

14163 Berlin

rainer.kuhlen@uni-konstanz.de

www.kuhlen.name

Dr. Rainer Kuhlen ist emeritierter Professor für Informationswissenschaft im Fachbereich Informatik und Informationswissenschaft an der Universität Konstanz und Sprecher des Aktionsbündnisses Urheberrecht für Bildung und Wissenschaft. 